\title{
Disminución del cortisol y mejora del estado de ánimo tras un programa psicoeducativo en cuidadores informales de personas con trastorno de la conducta alimentaria (TCA): un estudio preliminar. Decrease in cortisol and improvement in mood after a
psychotherapeutic program in informal caregivers of
people with eating disorders (ED): a preliminary study.
}

Fecha de recepción: 28/04/2014 Fecha de aceptación: 12/05/2015

\author{
Patricia Sariñana-González ${ }^{a}$, Nicolás Ruiz-Robledillo ${ }^{a}$, \\ Vanesa Pla ${ }^{b}$, Eugenia Moreno ${ }^{b}$, Luis Moya-Albiol ${ }^{a}$. \\ ${ }^{a}$ Departament de Psicobiologia. Universitat de València.

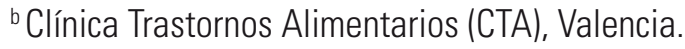

\section{resumen/alstract:}

El cuidado de un familiar con un trastorno de la conducta alimentaria (TCA) repercute negativamente en el cuidador, aumentando su percepción de estrés y deteriorando su calidad de vida. Los cuidadores informales de personas con TCA presentan mayores índices de ansiedad y depresión, y un peor estado de salud general. Se analizó la efectividad de un programa de intervención psicoeducativo en esta población, formado por 12 sesiones repartidas en 6 meses, y orientado a la reducción del estrés, la ansiedad y el estado de ánimo negativo. Se evaluaron los niveles de cortisol vespertino como marcador biológico de percepción de estrés, y la ansiedad y el estado de ánimo, antes y después de 3 sesiones del programa de intervención (pre-intervención, intermedia y post-intervención). Los resultados indicaron una mejora significativa del estado de ánimo y la ansiedad al finalizar el programa. Además, hubo una reducción de los niveles de cortisol vespertino en cada una de las sesiones evaluadas. En conclusión, el programa psicoeducativo utilizado fue efectivo para la mejora del estado de ánimo y la percepción de estrés. Futuros estudios deberían replicar estos resultados aumentando el tamaño muestral, incluyendo un grupo control e investigando los efectos positivos en los pacientes de TCA.

Caring for an offspring with an Eating Disorder (ED) has negative consequences for caregivers, increasing their stress perception and deteriorating their quality of life. Informal caregivers of people with an ED show higher levels of anxiety and depression, and worst general health. This study aimed to assess the effectiveness of a psychoeducational intervention program, oriented to the reduction of stress, anxiety and negative mood, in this population. The program was composed of 12 sessions, which were implemented over 6 months. Anxiety, negative mood, and afternoon cortisol levels before and after a 3 psychotherapeutic sessions (pre-intervention, mid-intervention and post-intervention) were assessed. Results showed a significant improvement in mood and anxiety after the intervention program. Furthermore, there was a significant reduction in afternoon cortisol levels in each of the evaluated sessions. In conclusion, the psychoeducational program applied was effective for improving mood and perceived stress. Future studies should replicate these results increasing the sample size, including a control group and analyzing the positive effects of this type of interventions in patients with EDs.

\section{palabras clave/keywords:}

programa psicoeducativo; cuidadores informales; trastorno de la conducta alimentaria (TCA); cortisol; estado de ánimo. Keywords: psychoeducational program; informal caregivers; eating disorders (ED); cortisol; mood. 
Los trastornos de la conducta alimentaria (TCA), entre los que se encuentran la anorexia y la bulimia nerviosas, se caracterizan fundamentalmente por una alteración en la ingesta alimentaria, relacionada con la distorsión de la propia imagen corporal (DSM-5; American Psychiatric Association, 2013). En el momento actual, este tipo de patologías constituyen un grupo de trastornos mentales que, debido a su carácter crónico y al deterioro progresivo que causan en los pacientes, presentan una gran relevancia social, económica y sanitaria, siendo un problema cuya prevalencia está aumentando considerablemente y cuyo comienzo se da cada vez a edades más tempranas (Aguinaga, Fernández y Varo, 2000). En este sentido, y en función de los estudios epidemiológicos realizados, la población de mayor riesgo son las mujeres jóvenes y adolescentes de países desarrollados o en vías de desarrollo (Contreras y González-Romo, 2010; Guía de Práctica Clínica sobre Trastornos de la Conducta Alimentaria, 2008; Peláez-Fernández, Labrador y Raich, 2005).

Dadas las características propias de los TCA y la sintomatología que los acompaña, el funcionamiento familiar se ve afectado de forma significativa. El hecho de cuidar de un familiar con una afección psicológica, cognitiva y/o conductual crónica, como pueden ser los TCA, implica en los cuidadores un afrontamiento diario de diversos desafíos asociados al cuidado (Ruiz-Robledillo y Moya-Albiol, 2012). De esta forma, el cuidador principal, al ser quien soporta la mayor parte de la sobrecarga física y emocional de los cuidados, suele presentar una seria afectación de la salud, tanto física como mental, además de un importante deterioro en su funcionamiento psicosocial, laboral y en su situación económica (BarreraOrtiz, Pinto-Afanador y Sánchez-Herrera, 2006; Vitaliano, Young y Zhang, 2004; Yates, Tennstedt y Chang, 1999). De este modo, estudios previos han puesto de manifiesto la existencia de altos niveles de malestar psicológico y ansiedad, así como numerosos problemas de salud en estos cuidadores (Coomber y King, 2012; Goddard, Macdonald y Treasure, 2011; Padierna et al., 2013; Whitney y Eisler, 2005; Winn et al., 2007; Zabala, Macdonald y Treasure, 2009). Dicha sintomatología, podría ser consecuencia directa de la disfunción familiar que conlleva el diagnóstico de TCA, llevándoles a padecer altos niveles de estrés, ya que se trata de una situación prolongada, en la que los cuidadores tienen que dedicar mucho tiempo y esfuerzo para adaptarse a las demandas del familiar con TCA y la situación de cuidado (Coomber y King, 2013; González, Padierna, Martín, Aguirre y Quintana, 2012; Martín, Padierna, Aguirre, Quintana, Hayas y Muñoz, 2011; Pérez-Pareja, Quiles, Romero, Pamies-Aubalat y Quiles, 2014).

Estudios previos en diversas muestras de cuidadores, como son aquellos de personas afectadas por la enfermedad de Alzheimer (Vedhara et al., 1999), esquizofrenia (González-Bono, de Andrés-García y Moya-Albiol, 2011), esclerosis múltiple (Vedhara et al., 2002) o personas con un trastorno de espectro autista (de Andrés-García, Sariñana-González, RomeroMartínez, Moya-Albiol y González-Bono, 2013; Ruiz-Robledillo y Moya-Albiol, 2013; Ruiz-Robledillo, González-Bono y Moya-Albiol, 2014), han mostrado una desregulación de diversos sistemas fisiológicos, fundamentalmente de aquellos relacionados con la respuesta de estrés y la salud, como el sistema endocrino, concretamente el eje hipotálamo-hipófisoadrenal (HHA) (Davis et al., 2004). Esta alteración está relacionada con el padecimiento de los altos niveles de estrés crónico, por lo que diversas investigaciones previas propusieron 
la disfunción de este sistema como un indicador fiable de las consecuencias que tiene sobre la salud y el funcionamiento psicológico de los cuidadores el hecho de prestar cuidados (de Andrés-García, Moya-Albiol y González-Bono, 2012; McEwen, 2003).

Por todo ello, atendiendo a los resultados obtenidos en estudios previos, se puso de manifiesto la necesidad de crear programas e intervenciones específicas dirigidas a cuidadores informales de personas con TCA, cuyo objetivo fundamental resida en la reducción de los problemas de salud y la percepción de estrés (Dare y Eisler, 2000; Salorio, Ruiz, Torres, Sanchis y Navarro, 1999; Scholz y Asen, 2001; Sepulveda et al., 2010; Tantillo, 2003, 2006; Vandereycken, Castro y Vanderlinden, 1991). En esta línea, una de las intervenciones mayormente utilizadas es el programa psicoeducativo de grupo basado en el modelo Maudsley, el cuál tiene como objetivo principal desarrollar habilidades de comunicación y de afrontamiento familiar adaptativas (Pla, Moreno, Fuentes-Durá y Dasí, 2014). A partir de dicho modelo y poniéndolo en práctica en dicha población, se obtuvieron resultados terapéuticos satisfactorios en diversos estudios, donde destacan la reducción del malestar psicológico y la mejora en la comprensión y la tolerancia hacia el TCA, además de un incremento de la autoeficacia del cuidado (Macdonald, Murray, Goddard y Treasure, 2011; Sepulveda, Whitney, Hankins y Treasure, 2008). Incluso en ciertos estudios, se ha observado como dicha mejoría se mantiene años después de la finalización de la intervención (Eisler et al., 2000; Goddard et al., 2011).

No obstante, hasta nuestro conocimiento, no se han llevado a cabo estudios que analicen de forma conjunta indicadores psicológicos y biológicos de estrés y salud para analizar la eficacia de este tipo de programas de intervención, en cuidadores informales de personas con TCA. Por ello, el objetivo principal de nuestro estudio consiste en evaluar la efectividad de una intervención psicoeducativa en la mejora del estado de ánimo, la ansiedad y los niveles de estrés en esta población. Este análisis ha sido llevado a cabo a través de indicadores psicológicos autoinfomados de ansiedad y estado de ánimo y parámetros biológicos de estrés, en concreto el cortisol vespertino. Basándonos en estudios previos llevados a cabo con otro tipo de cuidadores informales, esperamos obtener reducciones significativas en los niveles de cortisol vespertino durante las sesiones de intervención evaluadas (Ruiz-Robledillo, Sariñana-González, Pérez-Blasco, González-Bono y Moya-Albiol, 2014). Asimismo, esperamos observar mejorías significativas en la ansiedad y el estado de ánimo de los cuidadores a loa largo del programa de intervención (Macdonald, Hibbs, Corfield y Treasure, 2012; Pla et al., 2014; Ruiz-Robledillo et al., 2014).

\section{Metodología}

\section{Participantes}

La muestra estaba formada por padres $(n=2)$ y madres $(n=10)$ de personas con TCA, con una media de 48,58 \pm 1,19 años y un Índice de Masa Corporal (IMC) de 26,03 $\pm 1,24 \mathrm{~kg}$ / $\mathrm{m}^{2}$. Además, el 46,1\% de los cuidadores había realizado estudios superiores, el 23,1\% secundarios y el 30,8\% estudios primarios. Asimismo, el 53,8 \% de los participantes tenían una dedicación laboral de jornada completa, mientras que la otra mitad se encontraban empleados a media jornada o eran amas de casa. El 76,9\% de ellos no había participado con 
anterioridad en ninguna terapia psicológica, y sólo el 30,8\% contaba con antecedentes de familiares de primer grado de TCA.

La participación fue en todo momento voluntaria, sin ningún tipo de compensación económica, siendo requisito indispensable que los participantes fueran los cuidadores principales de un familiar de primer grado diagnosticado de algún TCA, según el DSM-IV-TR (2002), y compartieran el domicilio familiar. En lo referente a las horas semanales de contacto con la persona afectada por un TCA, el 53,8\% de los participantes superaban en más de 41 horas, el $15,4 \%$ cuidaban a sus familiares entre 21 y 30 horas semanales, el $23,1 \%$ entre 11 y 20 horas y el 7,7\% menos de 10 horas semanales.

Los participantes firmaron un consentimiento informado, acorde con las normas éticas de investigación con humanos (Declaración de Helsinki).

\section{Procedimiento}

El programa psicoeducativo se realizó en el Hospital de Día de la Clínica de Trastornos Alimentarios de Valencia (CTA) en colaboración con el Departament de Psicobiologia de la Universitat de València. La clínica CTA es un centro especializado en el diagnóstico, tratamiento y seguimiento de los TCA, que ofrece asistencia individualizada desde un enfoque multidisciplinar (psicológico, médico y nutricional). Tras planificar la investigación con la dirección del centro se informó a los cuidadores de la investigación a realizar de la posibilidad de participar voluntariamente en ella. Una vez que los participantes mostraron su disposición a colaborar se recogieron los datos socio-demográficos y de contacto necesarios.

\section{Programa de Intervención}

El programa psicoeducativo, ofertado de manera gratuita, consistió en una terapia de grupo para cuidadores informales de personas con TCA. Fue llevado a cabo por dos psicoterapeutas especialistas en TCA, con experiencia en técnicas de intervención grupal, que pertenecían a la plantilla laboral de la CTA. El grupo de cuidadores se reunió en 12 sesiones grupales de hora y media, con una periodicidad quincenal, durante un periodo aproximado de 6 meses. El programa, que constaba de 3 bloques, se desarrolló basándose principalmente en el modelo Maudsley (Treasure et al., 2008a, 2008b) y a partir de los talleres del programa original (Sepulveda, Lopez, Todd, Whitaker y Treasure, 2008), adaptando las sesiones en función de los objetivos que se pretendían alcanzar (véase Pla et al., 2014). En el bloque I de psicoeducación (sesiones 1, 2 y 3 ) se les explicaba a los familiares el concepto de TCA, las características que los definen y los factores multicausales que los generan. Se les enseñaban las etapas de cambio por las que van pasando tanto el paciente como los familiares durante el proceso de enfermedad. El objetivo era que los familiares pudieran identificar la sintomatología, practicando las habilidades de negociación frente a ésta, sin acomodarse a sus síntomas. En el bloque II (sesiones 4, 5, 6, 7, 8, 9, 10 y 11) se trabajaban las reacciones más frecuentes del cuidador de TCA, con el objetivo de que aprendieran a ejecutar una respuesta más eficaz. Se trabajaban las habilidades de comunicación, pretendiendo un cambio positivo en el lenguaje familiar y nociones básicas acerca del análisis funcional de conducta. El bloque III (sesión 12) se basaba en la prevención de recaídas; para lo que se 
les explicaba a los familiares en qué consiste una recaída, cómo poderla prevenir y cuáles son las cuestiones principales a tener en cuenta para poder paliar sus consecuencias. Cada sesión se estructuró de la siguiente manera: durante los 10 primeros minutos se realizaban ejercicios de relajación, autoestima, mindfulness, lectura de cuentos y ejercicios prácticos, para que los padres aprendieran a tomar conciencia de su estado emocional, de sus habilidades de resolución de problemas y reflexionasen sobre algunas cuestiones importantes que después se trabajarían en la sesión. A continuación, durante la hora y veinte minutos restante, se llevaba a cabo la parte psicoeducativa y de práctica de las habilidades y estrategias psicoterapéuticas objetivo de dicha sesión.

\section{Evaluación}

Durante el programa se evaluó el estado de ánimo, la ansiedad y el cortisol salivar vespertino (Csal) en tres sesiones: primera sesión (sesión pre-intervención), sexta sesión (sesión intermedia de la intervención) y última sesión (sesión post-intervención).

Para evaluar el Csal se recogieron cinco muestras de saliva en cada una de las sesiones evaluadas: dos muestras antes del comienzo de la sesión (pre y pre2), una inmediatamente después de finalizar la sesión (post) y, y otras dos muestras a los 15 y 30 minutos después (post15' y post30'). Además, cada participante completó dos cuestionarios, uno de ansiedad y otro de estado de ánimo, antes y después de cada sesión evaluada.

Dos horas previas a cada sesión y en el periodo de duración de la recogida de muestras se debían seguir fehacientemente las instrucciones facilitadas para preservar la calidad de las muestras de saliva: no ingerir comida ni ninguna sustancia psicoestimulante (alcohol, café, té, etc.), así como no cepillarse los dientes, fumar ni mascar chicle. En caso de realizar alguna de dichas actividades, se debía informar a los investigadores antes de comenzar la sesión. Una vez realizada la sesión psicoeducativa, los investigadores recogían las muestras, para posteriormente congelarlas a $-20^{\circ} \mathrm{C}$. El procedimiento seguido se presenta de forma resumida en la Tabla 1.

VER TABLA 1

\section{Variables}

\section{Cortisol Vespertino}

Las muestras de cortisol fueron recogidas mediante tubos de algodón salivettes (Sastedt, Rommersdolf, Germany), congeladas a $-20^{\circ} \mathrm{C}$ tras su recogida y analizadas mediante enzimoinmunoensayo. Todas las muestras de un mismo sujeto fueron analizadas por duplicado en el mismo ensayo obteniendo la media de los valores. El reactivo usado fue Coat-a-count Cortisol (DPC-Siemens Medical Solutions Diagnostics) con una sensibilidad de $1.4 \mathrm{nmol} / \mathrm{l}$. Los coeficientes de variación intra- e inter-ensayo fueron de 4.3 y $5.2 \%$, respectivamente. Todos los datos se muestran en nmol/l.

\section{Variables psicológicas estado}

Ansiedad Estado. Se evaluó a partir de la versión española del "State-Trait Anxiety Inventory" (STAI-E) (Spielberger, Gorsuch y Lushene, 1970; Seisdedos, 1988). Consta de 
Tabla 1. Tiempos de evaluación durante la aplicación del programa psicoeducativo.

\begin{tabular}{|c|c|c|c|}
\hline \multirow[b]{2}{*}{ Time } & $\begin{array}{c}\text { Evaluación } \\
\text { Pre- } \\
\text { intervención }\end{array}$ & $\begin{array}{l}\text { Evaluación } \\
\text { intermedia }\end{array}$ & $\begin{array}{c}\text { Evaluación post- } \\
\text { intervención }\end{array}$ \\
\hline & Pre-evaluación & Pre-evaluación & Pre-evaluación \\
\hline $\begin{array}{l}15 \\
\text { antes de } \\
\text { la } \\
\text { sesión }\end{array}$ & $\begin{array}{l}\text { Variables } \\
\text { psicológicas } \\
\text { estado } \\
\text { - STAI-E } \\
\text { - POMS } \\
\text { Cortisol } \\
\text { muestra pre-1 }\end{array}$ & $\begin{array}{l}\text { Variables } \\
\text { psicológicas } \\
\text { estado } \\
\text { - STAI-E } \\
\text { - POMS } \\
\text { Cortisol } \\
\text { muestra pre-1 }\end{array}$ & $\begin{array}{l}\text { Variables } \\
\text { psicológicas } \\
\text { estado } \\
\text { - STAI-E } \\
\text { - POMS } \\
\text { Cortisol } \\
\text { muestra pre-1 }\end{array}$ \\
\hline $\begin{array}{l}5 \text { ' antes } \\
\text { de la } \\
\text { sesión }\end{array}$ & $\begin{array}{l}\text { Cortisol muestra } \\
\text { pre-2 }\end{array}$ & $\begin{array}{l}\text { Cortisol muestra } \\
\text { pre-2 }\end{array}$ & $\begin{array}{l}\text { Cortisol muestra } \\
\text { pre-2 }\end{array}$ \\
\hline
\end{tabular}

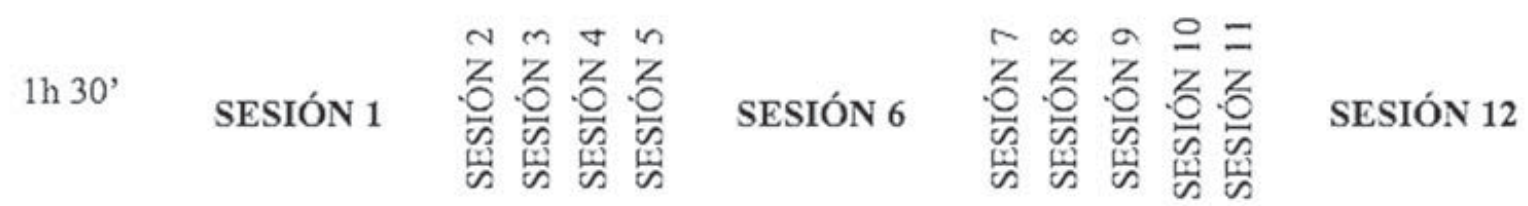

\begin{tabular}{|c|c|c|c|}
\hline & $\begin{array}{l}\text { Post- } \\
\text { evaluación }\end{array}$ & Post-evaluación & Post-evaluación \\
\hline $\begin{array}{l}0 \\
\text { después } \\
\text { de la } \\
\text { sesión }\end{array}$ & $\begin{array}{l}\text { Variables } \\
\text { psicológicas } \\
\text { estado } \\
\text { - STAI-E } \\
\text { - POMS } \\
\text { Cortisol } \\
\text { muestra post }\end{array}$ & $\begin{array}{l}\text { Variables } \\
\text { psicológicas } \\
\text { estado } \\
\text { - STAI-E } \\
\text { - POMS } \\
\text { Cortisol } \\
\text { muestra post }\end{array}$ & $\begin{array}{l}\text { Variables } \\
\text { psicológicas } \\
\text { estado } \\
\text { - STAI-E } \\
\text { - POMS } \\
\text { Cortisol } \\
\text { muestra post }\end{array}$ \\
\hline $\begin{array}{l}15 \\
\text { después } \\
\text { de la } \\
\text { sesión }\end{array}$ & $\begin{array}{l}\text { Cortisol muestra } \\
\text { post } 15^{\prime}\end{array}$ & $\begin{array}{l}\text { Cortisol muestra } \\
\text { post } 15 \text { ' }\end{array}$ & $\begin{array}{l}\text { Cortisol muestra } \\
\text { post } 15 \text {, }\end{array}$ \\
\hline $\begin{array}{l}30^{\prime} \\
\text { después } \\
\text { de la } \\
\text { sesión }\end{array}$ & $\begin{array}{l}\text { Cortisol muestra } \\
\text { post } 30^{\prime}\end{array}$ & $\begin{array}{l}\text { Cortisol muestra } \\
\text { post } 30^{\prime}\end{array}$ & $\begin{array}{l}\text { Cortisol muestra } \\
\text { post } 30^{\prime}\end{array}$ \\
\hline
\end{tabular}

veinte ítems donde el sujeto, a partir de una escala tipo Likert de 1 (nada) a 4 (mucho), debe describir su estado emocional en "ese momento exacto". Este instrumento cuenta con un coeficiente de fiabilidad de 0.62 .

Estado de ánimo. Se evaluó mediante la forma abreviada del Perfil de Estados de Ánimo (POMS) (McNair, Lorr y Droppleman, 1992), la cual se aplicó en base a los resultados obtenidos en el "estudio para construir una forma abreviada del Perfil de Estados de Ánimo (POMS)" de Fuentes, Balaguer, Meliá y García-Merita (1995). De las seis escalas y cin- 
cuenta y ocho adjetivos que forman la escala inicial, la forma adaptada está constituida por 29 adjetivos agrupados en cinco factores (Depresión, Vigor, Cólera, Fatiga y Tensión) y una puntuación total que se obtiene sumando las puntuaciones obtenidas en depresión, cólera, fatiga y tensión y restándole la puntuación del vigor. El sujeto debe contestar cómo se siente "ahora, en este momento" en una escala tipo Likert de 0 a 4 . Tiene una $\alpha$ de Cronbach de 0.80 .

\section{Análisis de datos}

Para el análisis de diferencias en el Csal vespertino antes y después de las sesiones de intervención evaluadas se emplearon pruebas de Friedman no paramétricas. Para ello, se compararon los niveles de Csal obtenidos en 4 momentos de medición: la media de las dos muestras pre-sesión (media pre) y los niveles obtenidos inmediatamente después de finalizar la sesión (post), y a los 15 (post15') y 30 minutos después de la misma (post30'). Las pruebas a posteriori consistieron en pruebas de rangos de Wilcoxon, con ajustes de Bonferroni para múltiples contrastes. En cuanto a las medidas de ansiedad y estado de ánimo, que se evaluaron en dos ocasiones (pre-sesión y post-sesión), en cada una de las sesiones evaluadas, también se emplearon pruebas de rangos de Wilcoxon, con ajustes de Bonferroni para múltiples contrastes. Para el análisis de las diferencias en los momentos pre y post sesión entre las tres sesiones de evaluación (pre-intervención, intermedia y post-intervención) se realizaron pruebas de Friedman no paramétricas, con pruebas a posteriori de rangos de Wilcoxon, con ajustes de Bonferroni para múltiples contrastes.

Todos los análisis estadísticos se realizaron mediante el software SPSS 21.0 (SPSS ${ }^{\circ}$ Statistics), considerándose como significativa toda probabilidad menor de 0.05. Los valores en tablas y figuras se expresaron en media \pm desviación típica.

\section{Resultados}

\section{Resultados por sesión}

En relación al Csal, se halló un efecto significativo del "momento" en todas las sesiones, $\chi 2(3)=25.778, \mathrm{p}=.000 ; \chi 2(3)=27.835, \mathrm{p}=.000 ; \mathrm{y} \chi 2(3)=29.672, \mathrm{p}=.000$; primera, intermedia y última sesión, respectivamente (ver figura 1). Para el análisis post hoc con los ajustes de Bonferroni, se estimó una significación de p<.008 para considerar las diferencias significativas. En las sesiones de evaluación se observaron diferencias significativas entre la medía de los momentos pre y todos los momentos post, $\mathrm{Z}=-3.059, \mathrm{p}=.002 ; \mathrm{Z}=-3.059$, $\mathrm{p}=.002 ; \mathrm{Z}=-3.061, \mathrm{p}=.002$, respectivamente, para la sesión pre-intervención; $\mathrm{Z}=-2.746$, $\mathrm{p}=.006 ; \mathrm{Z}=-3.059, \mathrm{p}=.002 ; \mathrm{y} \mathrm{Z}=-3.059, \mathrm{p}=.002$, respectivamente, para la sesión intermedia y $Z=-3.059, p=.002 ; Z=-3.059, p=.002 ; y Z=-3.059, p=.002$, respectivamente, para la sesión post-intervención. En la sesión intermedia y la sesión post-intervención también se hallaron diferencias entre el primer y último momento post (post 30'), $\mathrm{Z}=-2.845, \mathrm{p}=.004$ para la primera sesión y $Z=-3.062$, p=.002 para la última sesión. En todos los casos, los niveles de cortisol fueron más altos antes de la sesión que inmediatamente después de la misma y a los 15 y 30 minutos después. 
Figura 1. Descenso en los niveles de cortisol vespertino en cada una de las sesiones evaluadas (pre-intervención, sesión intermedia y post-intervención).

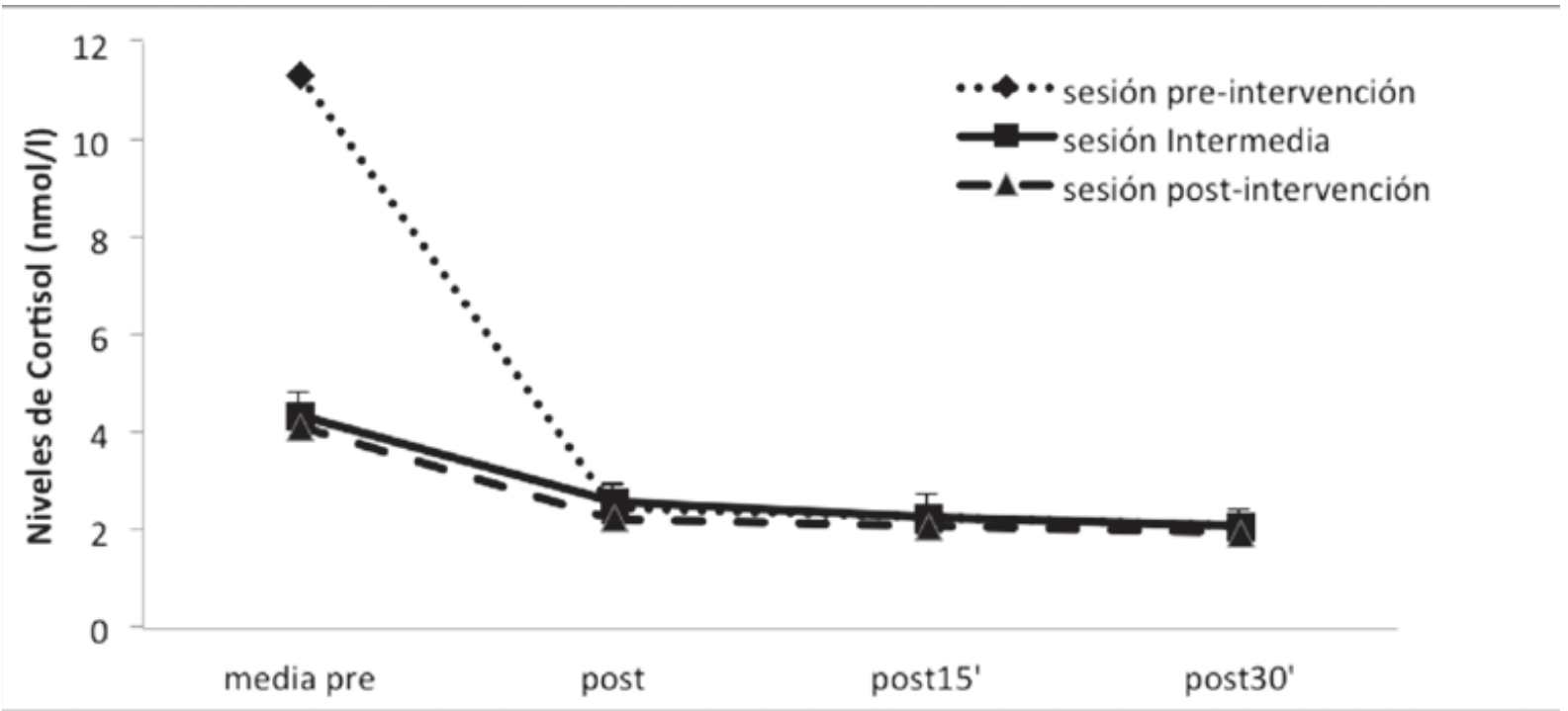

En relación a la ansiedad, se halló una diferencia significativa entre los momentos pre y post sesión en la sesión post-intervención, $Z=-2.938$, p=.003 (ver figura 2). En cuanto al estado de ánimo, también se encontraron diferencias significativas en la pre-intervención entre el momento pre y el post en las subescalas de depresión, $\mathrm{Z}=-1.947$, $\mathrm{p}=.051$, fatiga, $\mathrm{Z}=-2.251$, $\mathrm{p}=.024$, y tensión, $\mathrm{Z}=-2.593$, p=.010. Además, en la post-intervención se obtuvieron diferencias en las subescalas de depresión, $\mathrm{Z}=-2.132, \mathrm{p}=.033$, y vigor, $\mathrm{Z}=-2.552, \mathrm{p}=.011$ (ver tabla 3). Para todas la subescalas, excepto en el caso del vigor, las puntuaciones del momento post fueron menores que en el momento pre, por lo que se produjo una mejora del estado de ánimo en todos los casos.

Figura 2. Puntuaciones de los participantes en la ansiedad (STAI-E) $(* \mathrm{p}<.05)$.

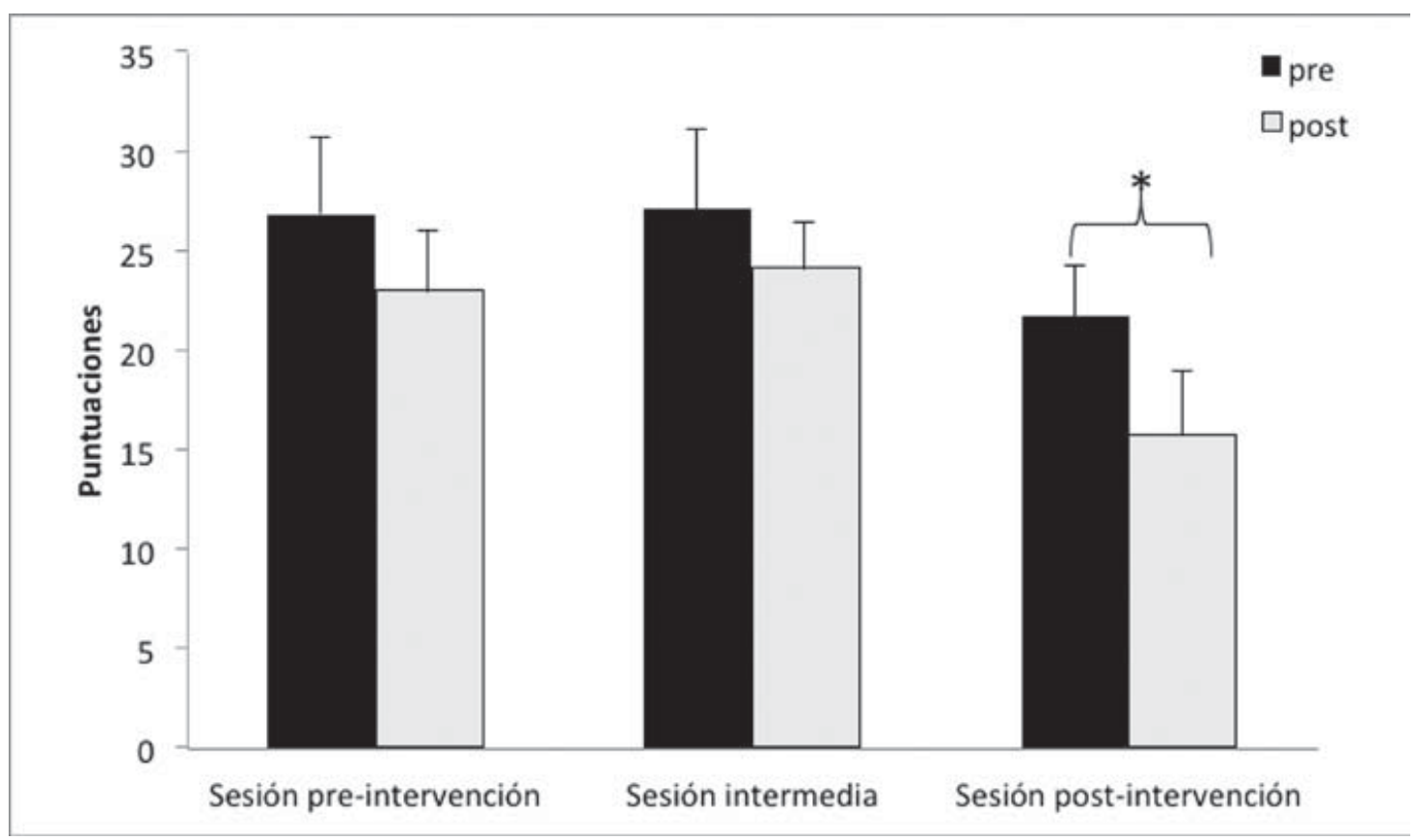




\section{Resultados del Tratamiento Global}

Respecto al estado de ánimo, se encontraron diferencias entre los niveles obtenidos en las evaluaciones pre-sesión de las tres sesiones de intervención en las subescalas de depresión, tensión y vigor, $\chi 2(2)=7.600, \mathrm{p}<.05 ; \chi 2(2)=6.884, \mathrm{p}<.05 ; \mathrm{y} \chi 2(2)=5.911, \mathrm{p}<.05$, respectivamente. En el caso de la depresión, las diferencias se encontraron entre la post-intervención y la sesión intermedia, $Z=-2.494, \mathrm{p}<.05$, mientras que en el caso de la tensión y el vigor, se hallaron diferencias significativas entre la post-intervención y las dos sesiones previas evaluadas, es decir respecto a la primera sesión y a la sesión intermedia, $\mathrm{Z}=-2.081, \mathrm{p}<.05$; y Z =2.674, $\mathrm{p}<.01$, para la tensión respectivamente, y $\mathrm{Z}=-2.712$, $\mathrm{p}<.01 ; \mathrm{y} \mathrm{Z}=-2.520$, $\mathrm{p}<.05$, para el vigor respectivamente. Excepto en el caso del vigor que aumentaron, las puntuaciones en las diferentes subescalas fueron disminuyendo a lo largo del programa de intervención.

Por lo que se refiere al momento post de evaluación, se encontraron diferencias significativas en las subescalas de depresión y vigor, $\chi^{2}(2)=6.588, \mathrm{p}<.05$; y $\chi^{2}(2)=7.682, \mathrm{p}<.05$, respectivamente. En concreto, las diferencias se observaron entre la última sesión y la intermedia, $\mathrm{Z}=-2.388, \mathrm{p}<.05 ; \mathrm{y} \mathrm{Z}=-2.586, \mathrm{p}<.01$, para depresión y vigor respectivamente. $\mathrm{Al}$ igual que en el momento pre de evaluación, la subescala depresión disminuyó a lo largo del programa de intervención, mientras que ocurrió lo contrario con la subescala vigor (Tabla 2).

Tabla 2. Puntuaciones de los cuidadores participantes en el estudio en el estado de ánimo. $(* \mathrm{p}<.05)$.

\begin{tabular}{|c|c|c|c|c|c|c|}
\hline & \multicolumn{2}{|c|}{$\begin{array}{c}\text { Evaluación } \\
\text { Pre-intervención }\end{array}$} & \multicolumn{2}{|c|}{$\begin{array}{c}\text { Evaluación inter- } \\
\text { media }\end{array}$} & \multicolumn{2}{|c|}{$\begin{array}{c}\text { Evaluación } \\
\text { Post-intervención }\end{array}$} \\
\hline & PRE & POST & PRE & POST & PRE & POST \\
\hline Depresión & $\begin{array}{l}7.08 \pm \\
1.95\end{array}$ & $4.50 \pm 1.49$ & $\begin{array}{c}6.67 \pm \\
1.97 *\end{array}$ & $\begin{array}{c}4.75 \pm \\
1.33 *\end{array}$ & $\begin{array}{c}2.83 \pm \\
1.11^{*}\end{array}$ & $\begin{array}{l}1.42 \pm \\
0.86^{*}\end{array}$ \\
\hline Vigor & $\begin{array}{c}11.58 \pm \\
1.71^{*}\end{array}$ & $\begin{array}{c}11.42 \pm \\
1.59\end{array}$ & $\begin{array}{c}10.58 \pm \\
1.77^{*}\end{array}$ & $\begin{array}{c}11.33 \pm \\
1.65^{*}\end{array}$ & $\begin{array}{c}13.92 \pm \\
1.42 *\end{array}$ & $\begin{array}{l}15.67 \pm \\
1.13^{*}\end{array}$ \\
\hline Cólera & $\begin{array}{l}5.83 \pm \\
2.12\end{array}$ & $4.00 \pm 1.74$ & $\begin{array}{l}6.92 \pm \\
2.27\end{array}$ & $\begin{array}{l}4.92 \pm \\
1.53\end{array}$ & $\begin{array}{c}2.50 \pm \\
1.03\end{array}$ & $\begin{array}{c}1.75 \pm \\
0.90\end{array}$ \\
\hline Fatiga & $\begin{array}{l}7.58 \pm \\
1.63\end{array}$ & $5.17 \pm 1.49$ & $\begin{array}{l}7.58 \pm \\
1.57\end{array}$ & $\begin{array}{l}5.75 \pm \\
1.33\end{array}$ & $\begin{array}{c}4.50 \pm \\
0.99\end{array}$ & $\begin{array}{l}3.92 \pm \\
0.85\end{array}$ \\
\hline Tensión & $\begin{array}{l}10.00 \pm \\
1.83^{*}\end{array}$ & $7.08 \pm 1.51$ & $\begin{array}{c}10.75 \pm \\
1.79^{*}\end{array}$ & $\begin{array}{c}7.83 \pm \\
1.49\end{array}$ & $\begin{array}{l}6.17 \pm \\
1.07^{*}\end{array}$ & $\begin{array}{c}4.17 \pm \\
0.92\end{array}$ \\
\hline
\end{tabular}

\section{Discusión}

Los resultados del presente estudio reflejan la efectividad del programa psicoeducativo aplicado en la reducción de la ansiedad y el estado de ánimo negativo en cuidadores de personas con TCA. En concreto, las puntuaciones en ansiedad y estado de ánimo fueron mayores en 
la primera sesión, mostrando una reducción progresiva a lo largo del desarrollo del programa de intervención. Asimismo, en cada sesión evaluada, se produjo una mejora del estado de ánimo y una reducción significativa de los niveles de cortisol. Todo ello evidencia la efectividad de este tipo de programas para la mejora del estado de ánimo y la percepción de estrés en particular y de la salud en general en cuidadores de TCA.

Los resultados obtenidos en nuestro estudio concuerdan con los de investigaciones previas en la misma población, en los que estrategias de intervención similares fueron efectivas en la mejora de la salud y la calidad de vida, reduciendo los niveles de ansiedad (Goddard et al., 2011; Grover et al., 2011). Asimismo, cuando se emplearon grupos de psicoeducación se halló una disminución del estrés, la depresión, los niveles de emoción expresada y el malestar psicológico, además de un aumento del bienestar autopercibido de la prestación de cuidados (Gutiérrez, Sepulveda, Anastasiadou y Medina-Pradas, 2014; Macdonald et al., 2011; Pla et al., 2014; Sepulveda et al., 2008; Uehara, Kawashima, Goto, Tasaki y Someya, 2001). En este sentido, el hecho de proporcionar herramientas útiles de afrontamiento y manejo de la situación de cuidado, así como facilitar información sobre la enfermedad y los objetivos del tratamiento de la persona cuidada, incrementa los sentimientos de autoconfianza disminuyendo de forma notable la percepción de sobrecarga y ansiedad. Ello resulta fundamental para conseguir una reducción significativa de la percepción de estrés y, por tanto, una mejora de su salud. Además de estos beneficios, es posible que la dinámica grupal en la que participaron permitiera que los cuidadores se encontraran en un ambiente positivo, donde poder dialogar con iguales, aprender y compartir experiencias y preocupaciones similares (Gutiérrez et al., 2014). Todo ello estaría relacionado con una reducción del estrés percibido y la ansiedad asociada a la situación de cuidado, a través de una percepción más adaptativa de su papel como cuidador y un mejor afrontamiento de la sintomatología del receptor de los cuidados y de la situación familiar.

Sin embargo, nuestro estudio va más allá, teniendo en cuenta que, hasta nuestro conocimiento, es el primero en emplear marcadores biológicos de estrés para el análisis de la efectividad terapéutica de un programa de intervención en esta población. Se ha empleado el cortisol vespertino, cuya reducción ha sido propuesta como un indicador fiable y sensible de la influencia de la relajación y la meditación sobre el funcionamiento del eje HHA y los niveles de estrés (Jevning, Wilson y Smith, 1978; Matousek, Dobkin y Pruessner., 2010; Ruiz-Robledillo et al., 2014). Por lo tanto, dicha reducción en los niveles de cortisol podría ser una consecuencia positiva de la puesta en práctica de ejercicios de relajación y meditación en cada una de las sesiones del programa psicoeducativo; basadas no sólo en la inducción de una estado de relajación en los participantes, sino también en tomar conciencia de sus pensamientos, emociones y sensaciones corporales, frente a eventos agradables y desagradables. En este sentido, nuestro estudio supone un avance en el conocimiento de los mecanismos biológicos subyacentes que pueden explicar la mejora terapéutica en el estado de salud y la percepción de estrés tras la aplicación del programa de intervención aplicado. Es decir, el hecho de contar con parámetros biológicos y autoinformados de salud supone una comprensión más fiable y global de la mejora en la percepción de los niveles de estrés y el estado de ánimo en los cuidadores de personas con TCA sometidos a un programa de intervención psicoeducativo. 
Aunque los resultados obtenidos en el presente estudio implican un avance significativo en la evaluación de la efectividad de un programa psicoeducativo en la mejora del estado de ánimo y la percepción de estrés, se deben tener en cuenta algunas limitaciones de este trabajo. Entre ellas cabe destacar la heterogeneidad de la muestra, al tratarse de cuidadores en etapas diferentes del tratamiento y en momentos diferentes en la evolución de la enfermedad de sus familiares. Además, la mayoría de las participantes fueron mujeres, aunque se ha de señalar que la disparidad del género en nuestros participantes se equipara a la realidad porcentual existente en la población de cuidadores informales (Ruiz-Robledillo y MoyaAlbiol, 2012), por lo que otorgaría a nuestro estudio de cierta validez ecológica. Otra de las limitaciones es el reducido tamaño de la muestra, si bien tal y como plantean en el estudio de Pla y cols. (2014) esto no es una desventaja en sí, ya que posiblemente al aumentar el tamaño muestral podríamos haber alcanzado la significación estadística en los contrastes que no la alcanzaron.

En conclusión, mediante la evaluación de parámetros psicológicos y de un marcador biológico relacionado directamente con el estrés, tal como es el cortisol vespertino, podemos corroborar las consecuencias positivas que puede tener una intervención terapéutica sobre la salud de cuidadores de TCA. No obstante, sería interesante incluir en futuros estudios un grupo control que no reciba dicha intervención al mismo momento, para poder establecer una causalidad directa en la efectividad del programa. Sin embargo, el hecho de evaluar tanto variables psicológicas como biológicas otorga al presente trabajo de un alto nivel de validez y fiabilidad para evaluar la efectividad de la intervención psicoeducativa sobre los cuidadores de TCA. Además, se podría ampliar la investigación a los receptores de los cuidados, ya que, como se ha propuesto previamente, los cuidadores informales pueden ejercer como factores de riesgo y de protección sobre la sintomatología y el funcionamiento adaptativo de los pacientes de TCA (Peláez-Fernández, Raich y Labrador, 2010), logrando una mejora en el proceso de recuperación, con el descenso de las conductas disfuncionales relacionadas con la alimentación y el malestar general (Goddard et al., 2011; Gutiérrez et al., 2014). Por último, también sería interesante conseguir un seguimiento de los cuidadores para examinar si los cambios se mantienen a más a largo plazo.

\section{Referencias}

American Psychiatric Association. (2013). Diagnostic and statistical manual of mental disorders, (DSM5®. American Psychiatric Pub.

Aguinaga, M., Fernández, L. J., \& Varo, J. R. (2000). Trastornos de la conducta alimentaria. Revisión y actualización. Anales del sistema sanitario de Navarra, 23(2), 279-292.

Barrera-Ortiz, L., Pinto-Afanador, N., \& Sánchez-Herrera, B. (2006). Evaluación de un programa para fortalecer a los cuidadores familiares de enfermos crónicos. Revista Salud Pública, 8(2), 141152.

Contreras, R. S., \& González Romo, Z. F. (2010). Estudio y análisis de los trastornos del comportamiento a través de las revistas científicas. Revisión bibliográfica de 2004 a 2008. Revista Electrónica de Psicología Iztacala, 13(1). 
Coomber, K., \& King, R. M. (2012). Coping strategies and social support as predictors and mediators of eating disorder carer burden and psychological distress. Social psychiatry and psychiatric epidemiology, 47(5), 789-796.

Coomber, K., \& King, R. M. (2013). A longitudinal examination of burden and psychological distress in carers of people with an eating disorder. Social psychiatry and psychiatric epidemiology, 48(1), 163-171.

Davis, L. L., Weaver, M., Zamrini, E., Stevens, A., Kang, D. H., \& Parker, C. R. (2004). Biopsychological markers of distress in informal caregivers. Biological Research for Nursing, 6(2), 90-99.

Dare, C., \& Eisler, I. (2000). A multi-family group day treatment programme for adolescent eating disorder. European Eating Disorders Review, 8(1), 4-18.

de Andrés-García, S., Moya-Albiol, L., \& González-Bono, E. (2012). Salivary cortisol and immunoglobulin A: Responses to stress as predictors of health complaints reported by caregivers of offspring with autistic spectrum disorder.Hormones and behavior, 62(4), 464-474.

de Andrés-García, S., Sariñana-González, P., Romero-Martínez, A., Moya-Albiol, L., \& Gonzalez-Bono, E. (2013). Cortisol response to stress in caregivers of offspring with autism spectrum disorder is associated with care recipient characteristics. Stress, 16(5), 510-519.

Eisler, I., Dare, C., Hodes, M., Russell, G., Dodge, E., \& Le Grange, D. (2000). Family therapy for adolescent anorexia nervosa: the results of a controlled comparison of two family interventions. Journal of Child Psychology and Psychiatry, 41(06), 727-736.

Fuentes, I., Balaguer, I., Meliá, J.L., \& García-Merita, M. (1995). Forma abreviada del Perfil de Estado de Ánimo (POMS). En E. Cantón (Comp.). V Congreso Nacional de Psicología de la Actividad Física y el Deporte, pp. 19-26. Valencia: Universitat de València.

Goddard, E., Macdonald, P., \& Treasure, J. (2011). An examination of the impact of the Maudsley Collaborative Care skills training workshops on patients with anorexia nervosa: A qualitative study. European eating disorders review, 19(2), 150-161.

Gonzalez-Bono, E., de Andrés-Garcia, S., \& Moya-Albiol, L. (2011). The cortisol awakening response in caregivers of schizophrenic offspring shows sensitivity to patient status. Anxiety, Stress \& Coping, 24(1), 107-120.

González, N., Padierna, A., Martín, J., Aguirre, U., \& Quintana, J. M. (2012). Predictors of change in perceived burden among caregivers of patients with eating disorders. Journal of affective disorders, 139(3), 273-282.

Grover, M., Williams, C., Eisler, I., Fairbairn, P., McCloskey, C., Smith, G., ... \& Schmidt, U. (2011). An off-line pilot evaluation of a web based systemic cognitive behavioral intervention for carers of people with anorexia nervosa. International Journal of Eating Disorders, 44(8), 708-715.

Gutiérrez, E., Sepulveda, A. R., Anastasiadou, D., \& Medina-Pradas, C. (2014). Programa de psicoeducación familiar para los trastornos del comportamiento alimentario. Behavioral Psychology / Psicología Conductual, 22(1), 131-147.

INSALUD (2008). Guías de Práctica Clínica en el Sistema Nacional de Salud. Madrid: Plan de Calidad para el Sistema Nacional de Salud del Ministerio de Sanidad y Consumo.

Jevning, R., Wilson, A. F., \& Smith, W. R. (1978). Adrenocortical activity during meditation. Hormones and Behavior, 10(1), 54-60.

Kyriacou, O., Treasure, J., \& Schmidt, U. (2008). Expressed emotion in eating disorders assessed via self-report: An examination of factors associated with expressed emotion in carers of people with anorexia nervosa in comparison to control families. International Journal of Eating Disorders, 41(1), 37-46.

Macdonald, P., Murray, J., Goddard, E., \& Treasure, J. (2011). Carer's experience and perceived effects of a skills based training programme for families of people with eating disorders: A qualitative study. European Eating Disorders Review, 19(6), 475-486. 
Macdonald, P., Hibbs, R., Corfield, F., \& Treasure, J. (2012). The use of motivational interviewing in eating disorders: A systematic review. Psychiatry research, 200(1), 1-11.

Martín, J., Padierna, A., Aguirre, U., Quintana, J.M., Las Hayas, C. \& Muñoz, P. (2011). Quality of life among caregivers of patients with eating disorders. Quality of life research, 20(9), 13591369.

Martín, J., Padierna, A., Aguirre, U., González, N., Muñoz, P., \& Quintana, J. M. (2013). Predictors of quality of life and caregiver burden among maternal and paternal caregivers of patients with eating disorders. Psychiatry research, 210(3), 1107-1115.

Matousek, R., Dobkin, P. L., \& Pruessner, J. (2010). Cortisol as a marker for improvement in mindfulness-based stress reduction. Complementary Therapies in Clinical Practice, 16(1), 13-19.

McEwen, B. S., (2003). Interacting mediators of allostasis and allostatic load: towards an understanding of resilience in aging. Metabolism, 52(10), 10-16.

McNair, D. M., Lorr, M., \& Droppleman, L. F. (1992). Manual for the Profile of Mood States. San Diego, California: EdITS/Educational and Industrial Testing Services. (Edición revisada).

Padierna, A., Martín, J., Aguirre, U., González, N., Muñoz, P., \& Quintana, J. M. (2013). Burden of caregiving amongst family caregivers of patients with eating disorders. Social psychiatry and psychiatric epidemiology, 48(1), 151-161.

Peláez-Fernández, M. A., Labrador- Encinas, F. J. \& Raich-Escursell, R. M. (2005). Prevalencia de los trastornos de la conducta alimentaria: consideraciones metodológicas. International Journal of Psychology and Psychological Therapy, 5(2), 135-148.

Peláez-Fernández, M. A., Raich-Escursell, R. M., \& Labrador- Encinas, F. J. (2010). Trastornos de la conducta alimentaria en España: Revisión de estudios epidemiológicos. Revista mexicana de trastornos alimentarios, 1(1), 62-75.

Pérez-Pareja, B., Quiles, Y., Romero, C., Pamies-Aubalat, L., \& Quiles, M. J. (2014). Malestar psicológico y emoción expresada en cuidadores de pacientes con un trastorno de la conducta alimentaria. Anales de psicología, 30(1), 37-45.

Pla, V., Moreno, E., Fuentes Durá, I., \& Dasí, C. (2013). Evaluación de un programa psicoterapéutico para cuidadores de pacientes con TCA: Estudio piloto. Informació Psicològica, 106, 28-40.

Ruíz-Robledillo, N. \& Moya-Albiol, L. (2012). El cuidado informal: una visión actual. Revista de Motivación y Emoción, 1, 22-30.

Ruiz-Robledillo, N., \& Moya-Albiol, L. (2013). Self-reported health and cortisol awakening response in parents of people with asperger syndrome: The role of trait anger and anxiety, coping and burden. Psychology \& health, 28(11), 1246-1264.

Ruiz-Robledillo, N., de Andrés-García, S., Pérez-Blasco, J., González-Bono, E., \& Moya-Albiol, L. (2014). Highly resilient coping entails better perceived health, high social support and low morning cortisol levels in parents of children with autism spectrum disorder. Research in developmental disabilities, 35(3), 686-695.

Ruiz-Robledillo, N., Sariñana-González, P., Pérez-Blasco, J., González-Bono, E., \& Moya-Albiol, L. (2014). A Mindfulness-Based Program Improves Health in Caregivers of People with Autism Spectrum Disorder: a Pilot Study. Mindfulness, 6(4), 767-777.

Salorio, P., Ruiz, M.E., Torres, A., Sanchis, F. \& Navarro, L. (1999). Psicoeducación familiar en grupo en los trastornos de la alimentación. Aplicación de un programa. Psiquiatria.com, 3(1).

Scholz, M. \& Asen, E. (2001). Multiple family therapy with eating disorders adolescents: Concepts and preliminary results. European Eating Disorders Review, 9(1), 33-42.

Seisdedos, N. (1988). Cuestionario de ansiedad estado-rasgo. Adaptación española. Madrid: TEA Ediciones.

Sepulveda, A. R., Lopez, C., Todd, G., Whitaker, W., \& Treasure, J. (2008). An examination of the impact of "the Maudsley eating disorder collaborative care skills workshops" on the well being of carers. Social Psychiatry and Psychiatric Epidemiology, 43(7), 584-591. 
Sepulveda, A.R., Whitney, J., Hankins, M. \& Treasure, J. (2008). Development and validation of an Eating Disorders Symptom Impact Scale (EDSIS) for carers of people with eating disorders. Health and quality of life outcomes, 6, 28-42.

Sepulveda, A. R., Todd, G., Whitaker, W., Grover, M., Stahl, D., \& Treasure, J. (2010). Expressed emotion in relatives of patients with eating disorders following skills training program. International journal of eating disorders, 43(7), 603-610.

Sepulveda, A.R., Graell, M., Berbel, E., Anastasiadou, D., Botella, J., Carrobles, J. \& Morande, G. (2012). Factors associated with emocional well-being in primary and secondary caregivers of patients with eating disorders. European eating disorders review, 20(1), 78-84.

Spielberger, R., Gorsuch, R., \& Lushene, R. (1970). STAI Manual for the State-Trait Anxiety Inventory 1970. Palo Alto, CA: Consulting Psychologists.

Tantillo, M. (2003) Eating disorders multifamily therapy group: capitalizing on the healing power of relationship. Eating disorders today, 2, 11-13.

Tantillo, M. (2006). A relational approach to eating disorders multifamily therapy group: moving from difference and diconnection to mutual connection. Families, systems \& health, 24(1), 82-102.

Treasure, J., Sepulveda, A., Macdonald, P., Whitaker, W., Lopez, C., Zabala, M., Kyriacou, O. \& Todd, G. (2008a). The assessment of the family of people with eating disorders. European Eating Disorders Review, 16(4), 247-255.

Treasure, J., Sepulveda, A.R., MacDonald, P., Whithaker, W., Lopez, C., Zabala, M., Kyriacou, O. \& Todd, G. (2008b). Interpersonal manteining factors in eating disorders: Skills sharing interventions for carers. International jounal of child and adolescent health, 1(4), 331-338.

Uehara, T., Kawashima, Y., Goto, M., Tasaki, S. I. \& Someya, T. (2001). Psychoeducation for the families of patients with eating disorders and changes in expressed emotion: a preliminary study. Comprehensive Psychiatry, 42(2), 132-138.

Vandereycken W., Castro, J. \& Vanderlinden, J. (1991). Anorexia y Bulimia. La familia en su génesis y tratamiento. Barcelona: Martínez-Roca.

Vedhara, K., Cox, N. K., Wilcock, G. K., Perks, P., Hunt, M., Anderson, S., ... \& Shanks, N. M. (1999). Chronic stress in elderly carers of dementia patients and antibody response to influenza vaccination. The Lancet, 353(9153), 627-631.

Vedhara, K., McDermott, M. P., Evans, T. G., Treanor, J. J., Plummer, S., Tallon, D., ... \& Schifitto, G. (2002). Chronic stress in nonelderly caregivers: psychological, endocrine and immune implications. Journal of psychosomatic research, 53(6), 1153-1161.

Vitaliano, P. P., Young, H. M., \& Zhang, J. (2004). Is caregiving a risk factor for illness?. Current Directions in Psychological Science, 13(1), 13-16.

Whitney, J., \& Eisler, I. (2005). Theoretical and empirical models around caring for someone with an eating disorder: The reorganization of family life and inter-personal maintenance factors. Journal of mental Health, 14(6), 575-585.

Whitney, J., Haigh, R., Weinman, J., \& Treasure, J. (2007). Caring for people with eating disorders: Factors associated with psychological distress and negative caregiving appraisals in carers of people with eating disorders. British Journal of Clinical Psychology, 46(4), 413-428.

Winn, S., Perkins, S., Walwyn, R., Schmidt, U., Eisler, I., Treasure, J., ... \& Yi, I. (2007). Predictors of mental health problems and negative caregiving experiences in carers of adolescents with bulimia nervosa. International Journal of Eating Disorders, 40(2), 171-178.

Yates, M. E., Tennstedt, S., \& Chang, B. H. (1999). Contributors to and mediators of psychological wellbeing for informal caregivers. The Journals of Gerontology Series B: Psychological Sciences and Social Sciences, 54(1), 12-22.

Yee, J. L., \& Schulz, R. (2000). Gender differences in psychiatric morbidity among family caregivers a review and analysis. The Gerontologist, 40(2), 147-164. 
Zabala, M. J., Macdonald, P., \& Treasure, J. (2009). Appraisal of caregiving burden, expressed emotion and psychological distress in families of people with eating disorders: A systematic review. European Eating Disorders Review, 17(5), 338-349. 